\title{
Monitoring Kinetic and Frequency-Domain Properties of Eyelid Responses in Mice With Magnetic Distance Measurement Technique
}

\section{S.K.E. Koekkoek, W. L. Den Ouden, G. Perry, S. M. Highstein and C. I. De Zeeuw} J Neurophysiol 88:2124-2133, 2002.

You might find this additional information useful...

This article cites 42 articles, 17 of which you can access free at:

http://jn.physiology.org/cgi/content/full/88/4/2124\#BIBL

This article has been cited by 4 other HighWire hosted articles:

LTP Regulates Burst Initiation and Frequency at Mossy Fiber-Granule Cell Synapses of

Rat Cerebellum: Experimental Observations and Theoretical Predictions

T. Nieus, E. Sola, J. Mapelli, E. Saftenku, P. Rossi and E. D'Angelo

J Neurophysiol, February 1, 2006; 95 (2): 686-699.

[Abstract] [Full Text] [PDF]

\author{
Response to Comment on "Cerebellar LTD and Learning-Dependent Timing of \\ Conditioned Eyelid Responses" \\ C. I. De Zeeuw, Y. Elgersma, H. C. Hulscher, B. R. Dortland, R. A. Hensbroek, T. J. H. Ruigrok \\ and S. K. E. Koekkoek \\ Science, April 9, 2004; 304 (5668): 211c-211c.
}

[Full Text] [PDF] Relative contributions of eyelid and eye-retraction motor systems to reflex and classically
conditioned blink responses in the rabbit

R. Leal-Campanario, J. A. Barradas-Bribiescas, J. M. Delgado-Garcia and A. Gruart

J Appl Physiol, April 1, 2004; 96 (4): 1541-1554.

[Abstract] [Full Text] [PDF]

Cerebellar LTD and Learning-Dependent Timing of Conditioned Eyelid Responses

S. K. E. Koekkoek, H. C. Hulscher, B. R. Dortland, R. A. Hensbroek, Y. Elgersma, T. J. H.

Ruigrok and C. I. De Zeeuw

Science, September 19, 2003; 301 (5640): 1736-1739.

[Abstract] [Full Text] [PDF]

Medline items on this article's topics can be found at http://highwire.stanford.edu/lists/artbytopic.dtl on the following topics:

Physics .. Magnetic Field

Neuroscience .. Conditioned Response

Education .. Associative Learning

Medicine .. Eyelids

Medicine .. Shock

Physiology .. Mice

Updated information and services including high-resolution figures, can be found at:

http://jn.physiology.org/cgi/content/full/88/4/2124

Additional material and information about Journal of Neurophysiology can be found at:

http://www.the-aps.org/publications/jn

This information is current as of November 14, 2006 . 


\title{
Monitoring Kinetic and Frequency-Domain Properties of Eyelid Responses in Mice With Magnetic Distance Measurement Technique
}

\author{
S.K.E. KOEKKOEK ${ }^{1}$ W. L. DEN OUDEN,${ }^{1}$ G. PERRY ${ }^{2}$ S. M. HIGHSTEIN ${ }^{2}$ AND C. I. DE ZEEUW ${ }^{1}$ \\ ${ }^{1}$ Department of Neuroscience, Erasmus University Rotterdam, 3000 DR, Rotterdam, The Netherlands; and ${ }^{2}$ Department \\ of Otolaryngology, Washington University School of Medicine, St. Louis, Missouri 63110
}

Received 16 May 2001; accepted in final form 12 April 2002

Koekkoek, S.K.E., W. L. Den Ouden, G. Perry, S. M. Highstein, and C. I. De Zeeuw. Monitoring kinetic and frequency-domain properties of eyelid responses in mice with magnetic distance measurement technique. J Neurophysiol 88: 2124-2133, 2002; 10.1152/jn.00396.2001. Classical eye-blink conditioning in mutant mice can be used to study the molecular mechanisms underlying associative learning. To measure the kinetic and frequency domain properties of conditioned (tone - periorbital shock procedure) and unconditioned eyelid responses in freely moving mice, we developed a method that allows adequate, absolute, and continuous determination of their eyelid movements in time and space while using an electrical shock as the unconditioned stimulus. The basic principle is to generate a local magnetic field that moves with the animal and that is picked up by either a field-sensitive chip or coil. With the use of this magnetic distance measurement technique (MDMT), but not with the use of electromyographic recordings, we were able to measure mean latency, peak amplitude, velocity, and acceleration of unconditioned eyelid responses, which equaled $7.9 \pm 0.2 \mathrm{~ms}, 1.2 \pm 0.02 \mathrm{~mm}, 28.5 \pm$ $1 \mathrm{~mm} / \mathrm{s}$, and $637 \pm 22 \mathrm{~mm} / \mathrm{s}^{2}$, respectively (means $\pm \mathrm{SD}$ ). During conditioning, the mice reached an average of $78 \%$ of conditioned responses over four training sessions, while animals that were subjected to randomly paired conditioned and unconditioned stimuli showed no significant increases. The mean latency of the conditioned responses decreased from $222 \pm 40 \mathrm{~ms}$ in session 2 to $127 \pm 6 \mathrm{~ms}$ in session 4, while their mean peak latency increased from $321 \pm 45$ to $416 \pm 67 \mathrm{~ms}$. The mean peak amplitudes, peak velocities, and peak acceleration of these responses increased from $0.62 \pm 0.02$ to $0.77 \pm$ $0.02 \mathrm{~mm}$, from $3.9 \pm 0.3$ to $7.7 \pm 0.5 \mathrm{~mm} / \mathrm{s}$, and from $81 \pm 7$ to $139 \pm 10 \mathrm{~mm} / \mathrm{s}^{2}$, respectively. Power spectra of acceleration records illustrated that both the unconditioned and conditioned responses of mice had oscillatory properties with a dominant peak frequency close to $25 \mathrm{~Hz}$ that was not dependent on training session, interstimulus interval, or response size. These data show that MDMT can be used to measure the kinetics and frequency domain properties of conditioned eyelid responses in mice and that these properties follow the dynamic characteristics of other mammals.

\section{IN T R O D U C T I O N}

Important insights have been obtained on the mechanisms underlying learning and memory by investigating the abilities of animals to condition their eyelid responses. Eyelid responses can either be conditioned in the standard way in which a conditioned stimulus (CS) such as a tone continues until the unconditioned stimulus (US) such as a corneal air-puff or an

Address for reprint requests: C. I. De Zeeuw, Dept. of Neuroscience, Erasmus University Rotterdam, P.O. Box 1738, 3000 DR, Rotterdam, The Netherlands (E-mail: dezeeuw@anat.fgg.eur.nl). electrical shock ceases, or one can acquire so-called traceconditioned eyelid responses in which the CS and US are separated by a trace interval. The main area for memory formation and storage underlying classical eye-blink conditioning is probably the cerebellum (Hesslow and Yeo 1998; Kim and Thompson 1997; McCormick and Thompson 1984), while the critical brain regions involved in trace conditioning also include higher structures such as the hippocampus (Disterhoft et al. 1999; Thompson et al. 1996).

Initially, most of the classical conditioning studies were done in rabbits and cats see e.g., (Attwell et al. 2001; Gruart et al. 1997; Steinmetz et al. 1992). These studies did not only elucidate which parts of the brain stem and cerebellum contribute to the formation and/or storage of conditioned responses, but they also provided insight into the framework of kinetic and frequency domain properties of both conditioned and unconditioned eyelid movements (Becker and Fuchs 1988; Evinger et al. 1991; Gruart et al. 1994, 2000a,b). Based on these findings it has been suggested that there must be a common oscillator that underlies eyelid movements (Domingo et al. 1997) and that the dominant frequency of this oscillator shows an inverse logarithmic relationship with the body weight of the type of species (Gruart et al. 2000a,b). To date the kinetic properties of eyelid movements in mice have not been described yet, and it is not known whether their eyelid oscillations follow the same relationship.

With the advent of transgenic technologies, it has become feasible to investigate the underlying mechanisms of eye-blink conditioning at the molecular level. Because the mouse is the only mammal of which embryonic stem cells are presently available that can be readily genetically manipulated for homologous recombination, this mammal is at present the preferred species to investigate the molecular mechanisms underlying learning and memory formation including those associated with eye-blink conditioning. For example, recent studies in mouse mutants were aimed at identifying the roles of mGluR1 and glial fibrillary acidic protein in the induction of cerebellar long-term depression and eye-blink conditioning (Aiba et al. 1994; Conquet et al. 1994; Shibuki et al. 1996). These studies have adopted the electromyographic (EMG) recording methods that were successfully applied for eye-blink conditioning in rabbits and cats. However, the question is

The costs of publication of this article were defrayed in part by the payment of page charges. The article must therefore be hereby marked "advertisement" in accordance with 18 U.S.C. Section 1734 solely to indicate this fact. 
whether this methodology is optimal for recording eyelid responses in mice. The much smaller size of the mouse and its facial musculature may make this preparation much more susceptible for picking up EMG activities of muscles that are not involved in the eye-blink response. For example, due to chewing or whisker movements during conditioning trials, EMG eye-blink recordings that are thought to result from activities in the musculus orbicularis oculi (MOO) may be contaminated by activities of larger surrounding muscles like the musculus masseter or musculus levator labii superior (MLLS). Such a spill-over of electrical signals may be particularly detrimental if one needs to obtain accurate recordings with an optimal spatiotemporal resolution. Moreover, for measuring position, velocity, or acceleration of conditioned and unconditioned eyelid responses, EMG recordings do have the disadvantages that they reflect muscle activities rather than directly the actual eyelid movement and that stimulus artifacts are picked up if the unconditioned stimulus (US) is delivered by an electrical shock.

An alternative could be to implant a search coil in the eyelid so as to detect an inductive current during eyelid rotation by placing the subject in a homogenous magnetic field. This method has been successfully applied for eye-blink conditioning in cats and humans (Becker and Fuchs 1988; DelgadoGarcia et al. 1990; Gruart et al. 2000a; Schicatano et al. 2002), but this approach is also unlikely to be optimal for mice as this method requires the subject to be restrained. Because mice are relatively sensitive for fixation, which can evoke various sorts of unwanted emotional, nonassociative, and even associative reactions during the process of eyelid conditioning, it appears particularly relevant for mice to do eye-blink conditioning in the freely moving preparation (see also following text).

We have therefore attempted to develop a method for eyeblink conditioning in mice that allows us to record their eyelid responses with a high spatiotemporal resolution in a freely moving state while applying conditioned and unconditioned stimuli without inducing artifacts in the recording. The basic principle is to generate a local magnetic field that moves with the animal and that is picked up by either a field sensitive chip or coil; we refer to this method as the magnetic distance measurement technique (MDMT). The spatiotemporal resolution of conditioned eyelid responses recorded in mice with the use of MDMT will be directly compared with that of EMG recordings, and the use of both methods in mice will be evaluated with the use of high speed video recordings. In addition, using MDMT we will provide for the first time the framework of kinetic and frequency domain properties of conditioned and nonconditioned eyelid movements of mice that can serve as a basis for further studies in transgenic mutants.

\section{METHODS}

\section{$M D M T$}

MDMT can be applied with the use of either two local coils or a magnet and a locally placed field-sensitive chip. The electronic circuitries of both possibilities are depicted in Fig. 1. In the first possibility, the magnetic field is generated by a transmitter coil (60 turns of $30-\mu \mathrm{m}$ enameled copper wire, $1.2 \mathrm{~mm}$ in diameter, $0.3 \mathrm{~mm}$ thickness, IET, Marly, Switzerland) placed above the upper eyelid, while a eyelid receiver coil (20 turns of $30 \mu \mathrm{m}$ enameled copper wire, $0.8 \mathrm{~mm}$ in diameter, $0.2 \mathrm{~mm}$ thickness, IET) implanted in the upper eyelid
A Transmitter coil

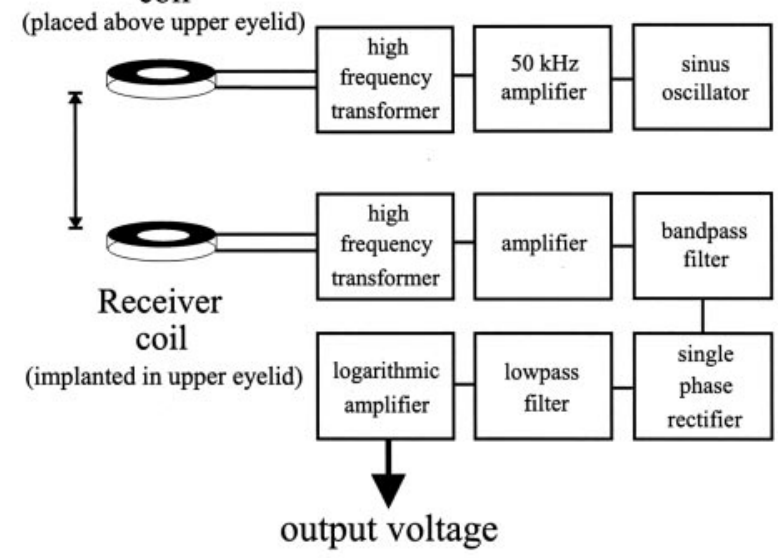

B GMR sensor

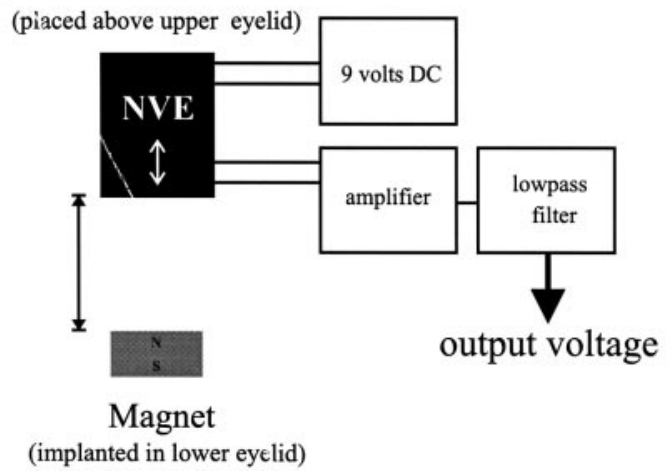

FIG. 1. Organization of magnetic distance measurement technique (MDMT) devices using either 2 minicoils or a GMR sensor chip with magnet. $A$ : the MDMT device with mini-coils is divided in a transmitter part and a receiver part, both of which are situated on the same print layout. The transmitter part consists of a current source $(100 \mathrm{~mA}, 50 \mathrm{kHz})$ and an amplifier that supplies the field generating coil. This coil is connected to the amplifier via a high-frequency transformer to prevent leakage of current to the coil. The small induced voltage of the receiver coil is fed into the receiver part via a high-frequency transformer after which the signal is amplified (40-90 dB). To reduce noise, the signal is band-pass filtered. Subsequently, the signal is single phase rectified, low-pass filtered and fed into a logarithmic amplifier, which delivers a signal proportional to the natural logarithm of the amplitude of the induced voltage. $B$ : the MDMT device with GMR sensorchip and a magnet is less complicated than the inductive system. A magnet provides an eyelid fixed constant magnetic field, which is detected by a GMR sensorchip. The chip consists out of a wheatstone bridge formed by 4 GMR resistors. Axis sensitivity (white arrow) is achieved by magnetically shielding 2 of the 4 resistors (NVE productsheet). The input voltage is provided by a $9-\mathrm{V}$ battery pack, which in this configuration provides a signal change of $30 \mathrm{mV}$ during eyelid closure. Amplification $(\times 100)$ and low-pass $(500 \mathrm{~Hz})$ filtering is done by an Axon Cyberamp 360

relays an induction signal, which is dependent on the changes of the mutual position of these two coils (Fig. 1A). The transmitter and receiver part of the system are situated on the same electronic print layout. The transmitter part consists of a sinus oscillator serving as a current source $(100 \mathrm{~mA}, 50 \mathrm{kHz})$ and an amplifier that supplies the field generating coil. The transmitter coil is connected to the amplifier via a high-frequency transformer to prevent leakage of current to the coil. The small induced voltage $(\approx 0.5 \mathrm{mV})$ in the receiver coil is fed into the receiver part via a high-frequency transformer after which the signal is amplified (40-90 dB). To reduce the noise of the signal it is band-pass filtered before it is single phase rectified, low-pass filtered , and fed into a logarithmic amplifier. Because the amplifier delivers a signal proportional to the natural logarithm of the amplitude of the 
induced voltage, the inductance in the receiver coil changes with displacement of the eyelid (Arts and Reneman 1980; Renterghem 1983). The data are captured with the use of a CED power1401 AD converter sampling at $1 \mathrm{kHz}$ coupled to an Axon Instruments Cyberamp 360 and analyzed off-line using custom written Matlab scripts.

In the second possibility, the magnetic field is generated by a magnet (neodymium iron borium; $0.8 \times 0.5 \times 0.2 \mathrm{~mm}$ in size, $<3 \mathrm{mg}$ weight, machined using 0.8-mm disk-type magnets (Wondermagnet: www.wondermagnet.com) implanted in the lower eyelid, while a giant magnetoresistive (GMR) sensorchip (NVE; AA004-MSOP) fixed above the upper eyelid picks up the strength of the magnetic field and delivers thereby a signal that depends on the position of the magnet, which has its north-south pole orientation across the width of the magnet (Fig. 1B). The application use of GMR sensors is comparable to that of so-called "Hall-effect" sensors (Hamiel et al. 1995; Korhonen 1991; Rodriguez et al. 2001). For details on GMR technology, see GMR technology sheet (NVE 2002). When a voltage is applied to the sensor, it returns a voltage that is determined by the strength of the magnetic field. Thus if the system is calibrated correctly, the voltage directly reflects the distance between the magnet and the sensor. System analysis of a dummy setup shows that following logarithmic conversion the sensor signal is linearly related to distances between 1.8 and $3.7 \mathrm{~mm}(<5 \%$ deviation). The signals are captured and analyzed as described $\sim$.

The surgical procedures to prepare the animals for MDMT were as follows. Adult male (6-10 wk) C57/B6 mice were anesthetized using an oxygenated mixture of nitrous oxide and halothane delivered through a cap fitting the snout $(n=18)$. A premade connector converted from a miniature connector (SamTec; www.samtec.com) to hold $\leq 12$ dual-pin connections on a $3 \times 8$-mm surface and a height of $3 \mathrm{~mm}$ was placed on the skull with the use of a pedestal of dental cement and 5 M1 screws. A coil or an in silicon embedded magnet was implanted in a pocket dissected in the eyelid. A suture wire glued to the coil or magnet was used to fixate this structure in the pocket. The pocket was closed with tissue glue. The transmission coil or sensor chip was carefully placed with the use of dental cement in an optimal position over the upper eyelid. For example, in case of the GMR sensor, optimal position was obtained when the distance between the magnet and sensor in the eyelid closed situation was $2 \mathrm{~mm}$ and the axis of sensitivity was aligned with the north-south axis of the magnet in the halfway closed position. Fully opened, fully closed and halfway opened positions were repetitively measured and stored for calibration purposes. Two insulated copper wires with a diameter of $30 \mu \mathrm{m}$ and $0.5-\mathrm{mm}$ tinned tips were placed underneath the skin to provide the US. The tips of the wires were located close to the lateral corner of the eyelids, one in the upper and one in the lower lid. The US electrode was connected to computer controlled stimulus isolation units (Dagan S910; www.dagan.com), which created biphasic constant current electrical shocks (30-ms, $166-\mathrm{Hz}$ pulses; max, $1 \mathrm{~mA}$ ). The strength of the stimulus was controlled by an UR based feedback mechanism so as to prevent potential induction of fear conditioning processes (Phillips and LeDoux 1992). This UR-based feedback was achieved by monitoring each UR and adjusting the stimulus strength to the minimal necessary to get full eyelid closure. The connecting cable including all wires was attached to a silicone mouse harness (Instech; www.instechlabs.com), which allowed the mice to move around during the experiment with relatively little discomfort since there is no torque or strain on the head. After the general surgery the animals were allowed to recover and adjust to their harnesses for $\geq 4$ days.

MDMT data were analyzed according to the following criteria. Eyelid movement $\geq 0.2 \mathrm{~mm}$ the mean +3 times SD of the $500-\mathrm{ms}$ pre-CS period was considered significant. Trials with significant activity in the pre-CS period were excluded, and trials with significant activity in the CS-onset to 75 -ms post CS-onset period were counted as startle responses. A conditioned response was counted if there was significant activity in the $75-\mathrm{ms}$ post CS-onset to US-onset time period. During CS alone trials, this period was extended with $200 \mathrm{~ms}$. Unconditioned blinks (40 trials) obtained from session 1 and conditioned blinks (40 trials) obtained from sessions 2-4 were used for response properties analysis. Velocity and acceleration traces were digitally computed. After low-pass filtering $(-3 \mathrm{~dB}$ cutoff at $75 \mathrm{~Hz}$ ), the first and second derivative of eyelid position traces were calculated. To estimate the relative strength of the different frequencies contained in eyelid responses. the power of the spectral density function of selected acceleration traces was calculated as described by Domingo et al. (1997).

\section{$E M G$}

Electromyographic recordings were obtained with the use of $30-\mu \mathrm{m}$ Teflon-coated $90 \% / 10 \%$ platinum/iridium wires (Advent Research Materials, Halesworth, U.K.) that were implanted in the MOO and MLLS $(n=6)$. The tips of the electrodes $( \pm 0.5 \mathrm{~mm})$ were stripped from their Teflon coating, tinned, and hooked, and their leads were led underneath the skin toward the head connector. As a guideline for placement of the MLLS electrodes, we used the middle of the second lateral row of whiskers. Signals from the EMG electrodes were fed into two AI402 preamplifiers connected to a Cyberamp 360 (both Axon Instruments; www.axon.com). The Cyberamp was attached to a CED (www.ced.co.uk) power1401.

EMG data were analyzed off-line using custom-written software (Spike2 and Mat-lab). The raw signals, which were sampled at 5,000 $\mathrm{Hz}$ for $1.5 \mathrm{~s}$, were rectified and the $100 \mathrm{~ms}$ before the CS presentation to $500 \mathrm{~ms}$ after the CS presentation were integrated over 5-ms bins (120 bins). The detection level was defined as the mean of the first 20 bins plus five times the SD. A constant value of 1 unit/bin was included as a conditioned response threshold (Chen et al. 1995). The trial was excluded if one of the bins in the control period exceeded the detection level. When the average unit count of the first six bins after CS onset was higher than the detection level, the response was considered to be a startle response and the trial was excluded. When at least one of the bins between bin 31 and the bin at which the UR started crossed the detection level, the response was marked as a conditioned response. The start of the first bin that crossed the level was taken as conditioned response onset, and the start of the bin with the highest value determined peak-latency. The mean value of the bins from conditioned response onset to the unconditioned response was used as a measure of conditioned response strength. In contrast to MDMT, the strength of the US was not controlled by a feedback mechanism because of the stimulus artifact.

The anesthesia and surgery were as described in the preceding text for MDMT except that the fifth screw of the pedestal was attached to pin 12 and functioned as a ground for the EMG recordings.

\section{Video}

A Kodak Ektapro HS motion analyzer was used to record the eye blinks with 1,125 frames/s (fps) at a spatial resolution of $256 \times 256$ pixels $(n=3)$. The camera, which produced 750 numbered bitmap files per blink (TIFF format), was triggered so as to start $250 \mathrm{~ms}$ before CS presentation and to stop $416 \mathrm{~ms}$ after CS onset (250-ms ISI). During the video recordings, the head of the mouse was fixed by attaching its connector to a male connector, which in turn was fixated to a restrainer that was placed on a small platform inside a shielded box. A circular cold light source provided sufficient lighting for the camera. Two light-emitting diodes (LEDs), which were visually shielded from the mice, functioned as visual indicators for the presence of the CS and US. The images were analyzed using customwritten software (Matlab). Each frame was color coded into 255 colors and smoothed by replacing the color value of each pixel with the average color value of that pixel and the eight immediately surrounding pixels. Of each recorded blink, the color range of the eyeball was determined, and each frame of that particular blink was 
subsequently transformed into a binary picture in which all pixels had a value of 0 unless its color value was within the predefined color range of the eyeball; in that case, it got a value of 1 . The percentage of visible eye surface was defined as the number of 1 's divided by the total number of pixels in the box area. This percentage plotted against frame time represented eyelid closure over time during the eye blinks. With the use of another analysis program custom written in Matlab, we produced a differential video sequence that was superimposed on the original images. This process resulted in a video of 50 frames (every 10th frame, starting at frame 200, ending at frame 700) where each frame showed the original image plus a color-coded image that represented changes in surface activity during the preceding $8.9 \mathrm{~ms}$ (10 frames at $1.125 \mathrm{fps}$ ).

The anesthesia and surgery were as described in the preceding text for MDMT except that the pedestal was adjusted for a head-fixed condition.

\section{Conditioning procedures}

The mice (C57/B6 males) were subjected to either a paired $(n=8)$ or a randomly paired procedure $(n=3)$. Both procedures lasted 4 days during each of which only one session was conducted. During one session, the subject received 100 trials grouped in 10 blocks. The trials were separated by a random inter-trial interval (ITI) in the range of $20-40 \mathrm{~s}$. The CS was a $10-\mathrm{kHz}$ tone with an intensity of $78 \mathrm{~dB}$ and a duration of $380 \mathrm{~ms}$ (ISI +30 -ms shock duration). The US electrical stimulus delivery was evaluated by analyzing the amplitude of the unconditioned response, and its strength was, if necessary, adjusted so as to obtain a full eyelid closure without a head-turn response. In the procedure of paired training each block consisted of one US-alone trial, eight paired trials, and one CS-alone trial (the 10th trial). After four sessions of paired training, the subject was allowed to rest for 1 day, followed by four sessions of extinction ( 1 session/day). In the extinction procedure each block consisted of one US-alone trial (1st trial) and nine CS-alone trials. In the randomly paired procedure, the US occurred randomly in the ITI.

To reduce experimental stress and unwanted associative or nonassociative responses, the boxes in which the training took place were sound proofed with the use of double walls filled with fine grained sand; the lighting, ventilation, and speakers were installed outside the electrically shielded inner layer; the mice were kept in their own cages during the training process in the box; and trials were performed only when the eyelid was fully opened and the mouse was not occupied with facial activities such as grooming or sniffing (during the experiment the eyelid and head were continuously monitored). A custommade script controlled the monitoring of eyelid and head, capturing of data, presentation of triggers and stimuli, and the handling of the file-system.

\section{RES ULTS}

Kinetic and frequency domain properties of conditioned and nonconditioned eyelid responses as determined with the use of $M D M T$

The data given below have been obtained with the use of both coil-MDMT and chip-MDMT. These two approaches differed somewhat on some practical issues such as the stability of implantation in the eyelid (coil $>$ magnet) and the voltage signal-to-noise ratio (magnet-chip system $>$ coil), but they did not show any significant difference with respect to any of the experimental data. This similarity showed that the basic technical principle of MDMT on which both approaches are based is applicable in a reliable fashion and that the results can be pooled as done in the following text. Eleven unrestrained C57/B6 mice were conditioned to a tone (CS) with the use of a small electrical shock as the US. The ISI between the CS and US was $350 \mathrm{~ms}$. The average percentage of conditioned responses in the CS alone trials gradually increased from $6 \pm$ $2.6 \%$ (mean $\pm \mathrm{SE}$ ) to $78 \pm 10.1 \%$ over 4 days of training (see T-1 to T-4 in Fig. 2A). In contrast, none of the animals $(n=3)$ that were subjected to randomly paired CS and US for control showed significant increases in their percentages of conditioned responses. The conditioned responses in the animals that were trained with paired stimuli could be extinguished to pretraining levels over a period of four sessions (see E1-E4 in Fig. 2A).

The unconditioned blink responses of session 1 (i.e., T-1) showed a mean latency of $7.9 \pm 0.2$ (SE) ms (40 trials; Fig. $2 B$, left). The mean peak amplitude, velocity, and acceleration of these responses were $1.2 \pm 0.02 \mathrm{~mm}, 28.5 \pm 1 \mathrm{~mm} / \mathrm{s}$, and $637 \pm 22 \mathrm{~mm} / \mathrm{s}^{2}$, respectively. There was a significant linear relationship ( $r=0.92 ; P<0.0001)$ between the peak velocity and the maximum amplitude of the evoked movement (Fig. $3 A$ ). The mean peak amplitudes of conditioned responses of sessions $2-4$ were $0.62 \pm 0.02,0.78 \pm 0.01$, and $0.77 \pm 0.02$ (SE) mm (40 trials for each session, CS-alone trials), respectively (Fig. 2B, right), while their mean peak velocities increased from $3.9 \pm 0.3 \mathrm{~mm} / \mathrm{s}$ in session 2 to $7.7 \pm 0.5 \mathrm{~mm} / \mathrm{s}$ in session 4. Accordingly, the mean peak acceleration increased from $81 \pm 7$ to $139 \pm 10 \mathrm{~mm} / \mathrm{s}^{2}$. The mean latency of the responses decreased from $222 \pm 40 \mathrm{~ms}$ in session 2 to $127 \pm 6 \mathrm{~ms}$ in session 4, while the mean peak latency of the conditioned responses increased from $321 \pm 45$ to $416 \pm 67$ ms. Although the relationship between conditioned response peak velocity and peak amplitude was significant $(P<0.0001$, Fig. $3 B$ ), the correlation coefficient was lower than that of the unconditioned responses $(r=0.63)$.

Oscillatory components were visible in most of the responses. Power spectra of acceleration records of both unconditioned and conditioned responses showed a dominant peak at a frequency of $\sim 25 \mathrm{~Hz}$ (for unconditioned responses, a mean of $25 \pm 0.8 \mathrm{~Hz}$; for conditioned responses, a mean of $24 \pm 0.7$ $\mathrm{Hz}$; Fig. 2B). These dominant frequencies were not influenced by the number of the session or by the length of the ISI (for control 3 and 4 additional animals were tested with ISIs of 250 and $450 \mathrm{~ms}$, respectively). Thus the longer conditioned responses that occurred in the later training sessions were generated by an increase in the number of waves but not by a change in the dominant frequency.

\section{EMG recordings}

To find out to what extent the data obtained with MDMT differ from those obtained with EMG and whether EMG signals from the MOO can be contaminated with signals originating from surrounding muscles, we recorded simultaneously with separate EMG electrodes signals from the MOO and MLLS of adult male C57/B6 mice $(n=6)$ during eye-blink conditioning. The response curves are shown in Fig. 4A. Over 4 consecutive days of training (T-1 to T-4), the percentage of significant responses recorded on the MOO electrodes increased to a level of $75 \%$, whereas that of the MLLS electrodes increased to an average of $36 \%$. The SDs of these percentages were significantly higher than those obtained with the use of MDMT ( $P<0.02$; Student's $t$-test). Moreover, the percentage of conditioned responses on $\mathrm{T}-1$ was significantly higher than 


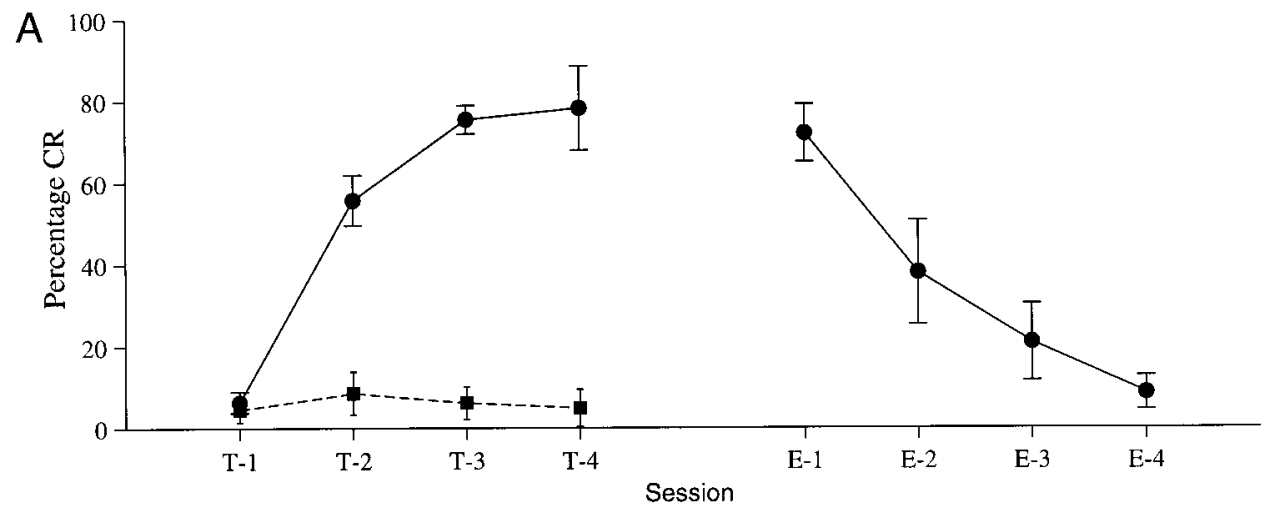

B
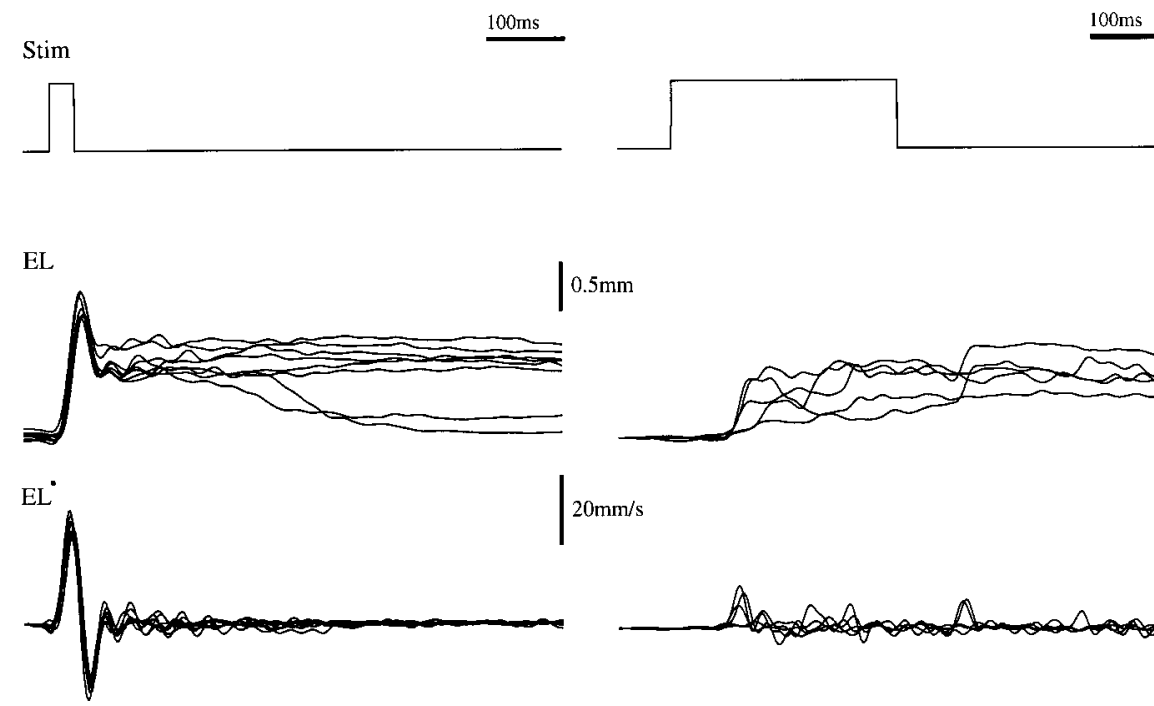
$20 \mathrm{~mm} / \mathrm{s}$

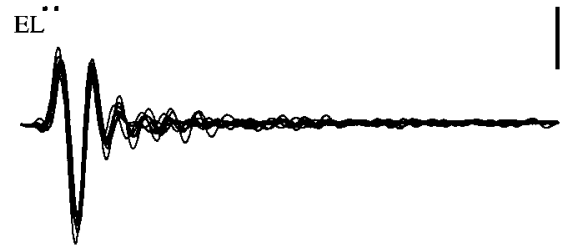

$500 \mathrm{~mm} / \mathrm{s}^{2}$
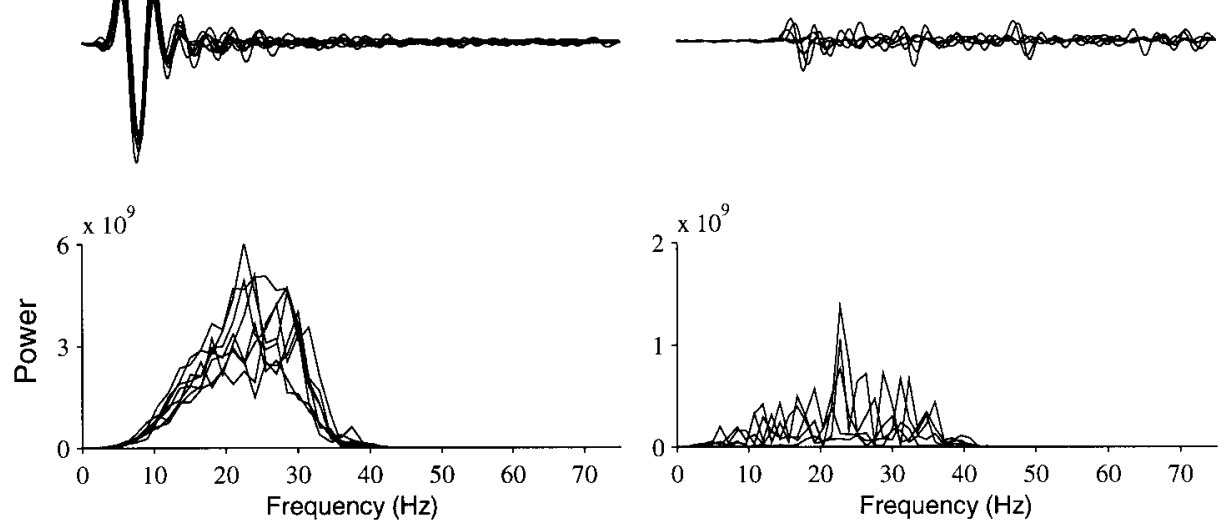

FIG. 2. Eyeblink conditioning with the use of MDMT. A: the percentages of the conditioned responses increase during normal paired training $(\bullet, \mathrm{T}-1$ to $\mathrm{T}-4)$ but not during randomly paired training (ם), while the rate decreases during the extinction period (E-1 to E-4). Values are group averaged response counts; error bars indicate SE. B: examples of raw eyelid position traces are depicted as well as their 1st and 2nd derivatives, stimulus trace and power spectrum. Left: 8 unconditioned response (UR) recordings obtained from UR-only trials in session 4 of an animal. The UR traces all show a high similarity resulting in a clear response profile and uniform spectrum shape. Right: 5 conditioned response (CR) traces obtained from conditioned stimulus (CS)-alone trials in session 4 of the same animal. Each CR depicted here shows a different shape. The CRs can have different shapes but usually reach peak amplitude close to the end of the interstimulus interval (ISI). Due to the different shapes the power spectra are not so uniform when compared with the UR but the dominant frequency remains close to $25 \mathrm{~Hz}$, suggesting that a common oscillator underlies eyelid motor system behavior. The eyelid responses were conditioned using an ISI of 350. that obtained with the use of MDMT $(P<0.01$; Student's $t$-test). The percentage of responses recorded on the MOO electrodes could be reduced to baseline levels after 4 days of extinction, whereas that of the MLLS showed only a minor reduction (Fig. 4A, left). The mean sizes of the MOO responses increased and decreased consistently over consecutive days of training and extinction, respectively, while those of the MLLS already diminished to minimal levels on day 4 of the training (Fig. 4A, right). All attempts to measure frequency and time domain properties from EMG traces resulted in a large variation in the data. Thus several data points obtained with the use of EMG recordings differed from those obtained with MDMT recordings, and the MLLS electrodes picked up a substantial percentage of responses. Together these observations raise the possibility that snout responses contaminate the eyelid recordings.

\section{Evaluation with the use of high-speed video imaging}

To find out whether snout movements can indeed contaminate eye-blink recordings in mice and to what extent the MDMT and/or EMG recordings reflect the actual kinetics of the eyelid movement, we evaluated these methods with the use 

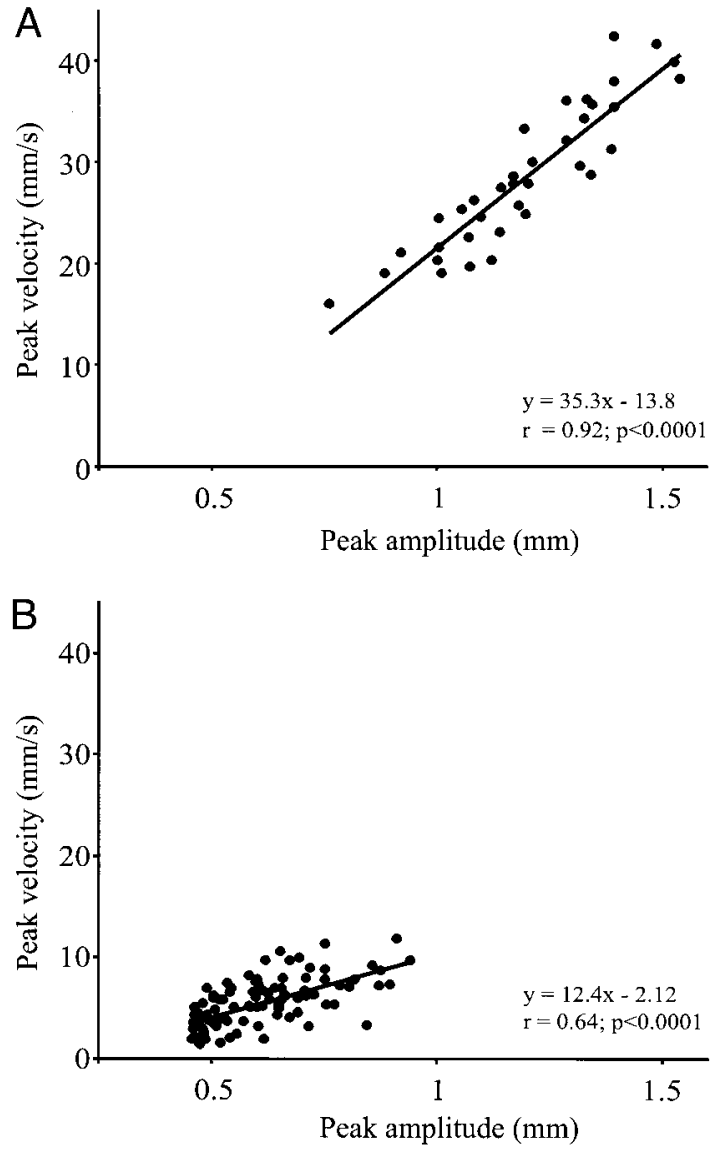

FIG. 3. UR and CR peak velocity as a function of maximum amplitude. $A$ : UR peak velocity is plotted against peak amplitude. There was a significant linear relationship indicating that larger responses are made by increasing the peak velocity. $B$ : the same relationship but now for conditioned responses showed a much lower but still significant correlation; this is suggestive for external (outside the classical eyeblink conditioning pathway) influences on the eyelid motor system during conditioning trials.

of a high-speed video system by recording and analyzing conditioned and unconditioned eyelid responses in restrained mice with all three methods simultaneously $(n=3)$. The spatiotemporal resolution of the video system, which operated at $1,125 \mathrm{fps}$ for $256 \times 256$ pixels, appeared sufficient to reliably detect and visualize simultaneously both eye-blink movements and other facial muscle responses (Fig. $4 B$ ). In $37 \%$ of all trials, the video analysis showed a facial response without any eyelid movement. Yet, in $43 \%$ of these cases, the EMG recordings of the "MOO" detected a positive eyelid response, whereas none of the MDMT recordings showed a significant eyelid movement. These data indicate that noneyelid facial movements can occur in the process of eye-blink conditioning in mice and that the muscle activities underlying these movements can contaminate EMG recordings of the MOO. This contamination is most likely due to incorrect electrode placing or wandering electrodes, even if they have been carefully placed. The superior detection of the actual displacement of the eyelid with the use of MDMT was particularly evident in trials in which irregular movements occur. Figure 5 shows such a trial in which the MDMT recordings correspond to those of the video recordings, while the EMG recordings diverge. The mean peak latency obtained from the MDMT recordings (179 $\pm 10.2 \mathrm{~ms})$ was comparable with that calculated from the video traces $(175 \pm 12.9 \mathrm{~ms})$, whereas the mean peak latency of the EMG signals was significantly lower (EMG: $125 \pm 18.9 \mathrm{~ms} ; P=0.02$ Student's $t$-test). Thus the fact that MDMT, but not EMG, produces results comparable to those observed with high-speed video recordings demonstrates that MDMT is suited for determination of eyelid position in mice and thus for investigation of the kinetic properties of conditioned and unconditioned responses.

Interestingly, the percentage of discrete eyelid conditioned responses in the mice that had to be restrained for the stability of the video recordings was much smaller than could be expected from the recordings in the freely moving mice that have been described above. For example, in trial 300-400, the average percentage of discrete eyelid responses was only $9 \pm$ $3 \%$. These reductions held true for both numbers obtained with MDMT recordings and with EMG recordings raising the possibility that stress induced by fixation of the head and body can impair classical eye-blink conditioning. In contrast, during the same trials the percentage of nondiscrete responses was on average $87 \pm 5 \%$. In $61 \pm 4 \%$, this nondiscrete response included an eyelid response. For example, Fig. $4 B$ (3rd row) shows an early nondiscrete component without eyelid activity accompanied by a late eyelid only component.

\section{I S C U S S I O N}

We present a novel method, MDMT, which allows continuous recording of eyelid position over time in freely moving mice. For the first time, the response kinetics of conditioned and unconditioned eyelid responses in mice were measured and documented. In the following text, we discuss the kinetic and oscillatory properties of eyelid responses in mice, factors in the setup that may influence the rate of conditioning in mice, and potential future applications of MDMT.

As measured with the use of MDMT, the average percentage of conditioned responses in CS alone trials of freely moving mice gradually increase to $78 \%$ over 4 days of training, while they decrease to baseline level within 4 days of extinction. These percentages agree well with those obtained with the use of EMG recordings (present study; Chen et al. 1996; Conquet et al. 1994; Kishimoto et al. 2001). Yet, our combined MDMTEMG-video recordings demonstrated that EMG recordings in restrained mice can pick up a substantial number of falsepositive conditioned responses due to snout movements, whereas MDMT recordings will not detect a conditioned eyelid response when the eyelid is not moving. This discrepancy in response counts suggests that when EMG electrodes are placed in the eyelid of a mouse they do not only pick up false-positive responses from surrounding musculature but also false-negative responses in cases of MOO only responses. In mice, the MOO is a thin muscle, which is much thinner than the actual eyelid and covers the bottom of the eyelid following the curve of the eyeball. Thus as one needs EMG electrodes of a particular minimum size with a particular minimum stiffness to pick up sufficient signals over $\geq 4$ days of training, it appears impossible to place these electrodes perfectly and permanently in the optimal site of the small MOO muscle of a mouse.

The measurements done with the use of MDMT, but not EMG, provided stable and reliable data on the kinetics of eyelid movements in mice. The mean latency of their unconditioned eyelid responses equaled $7.9 \mathrm{~ms}$, which is comparable 
A
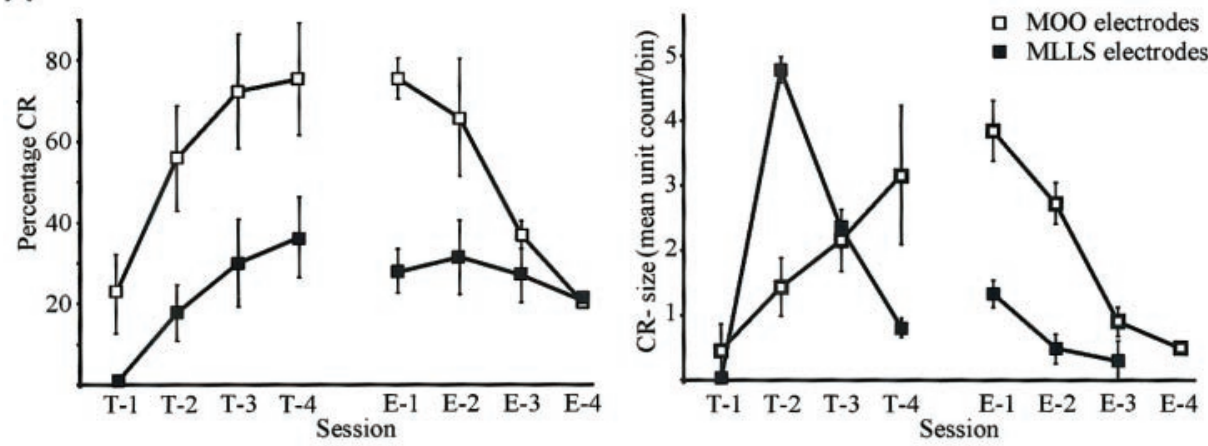

B

First paired trial in naive animal
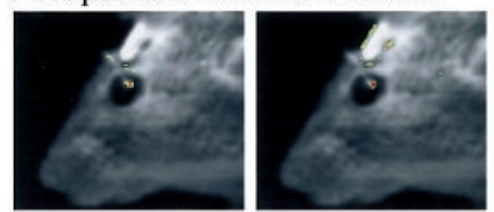

During training, paired trial
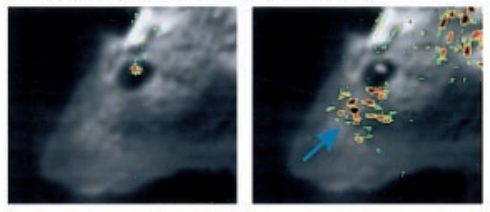

After training, CS-alone trial
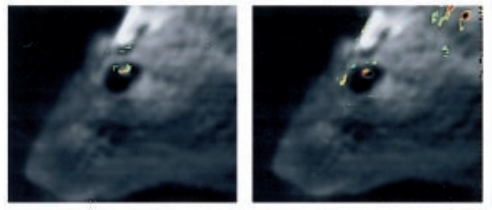

$\mathrm{T}=0$

$\mathrm{T}=97$

to values described for rabbit (Quinn et al. 1984) and compatible with a disynaptic pathway mediating the UR (van Ham and Yeo 1996). The mean velocity $(28.5 \mathrm{~mm} / \mathrm{s})$ and acceleration $\left(637 \mathrm{~mm} / \mathrm{s}^{2}\right)$ that we found for eyelid movements in mice cannot be directly compared with those previously obtained in rabbits, rats and cats (see e.g., Gruart et al. 1994) because we directly recorded changes in distance while our colleagues measured coil rotations. However, the ratio between peak amplitude and peak velocity and the ratio between peak velocity and peak acceleration in mice (1:24 and 1:22) agreed well with the same values that can be obtained from rabbits (1:17 and 1:18) (Gruart et al. 2000b). In addition, the correlation coefficient of the observed linear relationship between unconditioned response peak amplitude and peak velocity in mice $(r=0.92)$ is similar to that in rabbit $(r=0.91)$. Thus as described by Gruart and colleagues (2000b) for eyelid responses in rabbits, larger lid responses in mice may be predominantly achieved by increasing the velocity of the movement. On the other hand, it should be noted that species differences can occur as the ratios mentioned in the preceding text for mice and rabbits diverge from those in cats (1:43 for the ratio between peak amplitude and peak velocity and 1:52 for the ratio between peak velocity and peak acceleration) (Domingo et al. 1997).

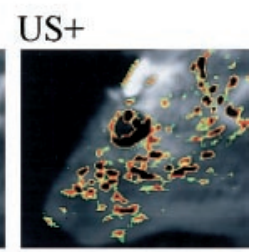

\section{$\mathrm{US}+$}

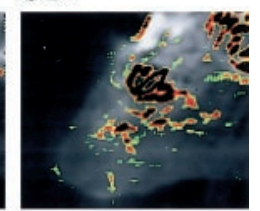

US-

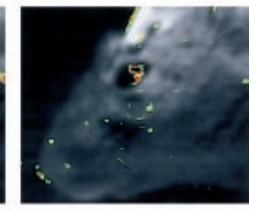

$\mathrm{T}=257$

FIG. 4. Nondiscrete facial co-contractions displayed by video recordings and confirmed by electromyography (EMG). A: the occurrence of nondiscrete facial responses in freely moving mice was quantified by recording EMG activities of both the musculus levator labii superior (MLLS) and musculus orbicularis oculi (MOO) during conditioning. Left: that the averaged percentage of the conditioned responses of the MLLS increased to $36 \%$ over 4 consecutive days of training (T-1 to T-4), while that of the MOO increased to a normal level of $75 \%$. The percentage of conditioned responses recorded on the MOO electrodes could be reduced to baseline levels following 4 days of extinction, while that of the MLLS showed only a minor reduction. Right: the mean size of the CR of the MOO responses increased consistently over consecutive days of training, whereas those of the MLLS diminished to minimal levels on day 4 . Thus the musculature of the snout of a freely moving mouse does show conditioning behavior with the use of an electrical shock at the eye corner as the US, but the amplitudes of its conditioned responses diminish over time. $B$ : differential video frame analyses during the training confirmed that during the training process co-contractions start to occur in the facial musculature of the snout (blue arrows) indicating that aspecific responses can occur that can contaminate the counts and the response properties of conditioned eyelid responses (red arrows).

The kinetic parameters of conditioned responses in mice depended on the number of the training session. Over the trials, the mean peak latency, amplitude, velocity, and acceleration of the conditioned responses increased to $416 \pm 76 \mathrm{~ms}, 0.77 \pm$ $0.02 \mathrm{~mm}, 7.7 \pm 0.5 \mathrm{~mm} / \mathrm{s}$ and $139 \pm 10 \mathrm{~mm} / \mathrm{s}^{2}$, respectively, while mean onset latency of the responses decreased to $127 \pm$ $6 \mathrm{~ms}$. Peak latency and onset latency were comparable to those found in rabbits (Gruart et al. 2000b). Using an ISI of $250 \mathrm{~ms}$, the onset latency of well-trained rabbits equaled $\sim 130 \mathrm{~ms}$, and the peak latency of their conditioned responses also occurred somewhat after the ISI. The conditioned responses in mice also resembled those in cats and rabbits in that the larger conditioned responses were generated by changing the amplitude and/or number of waves (Domingo et al. 1997; Gruart et al. 2000a,b). Moreover, the differences between the conditioned and unconditioned responses of mice were similar to those of rabbits and cats (see e.g., Gruart et al. 2000b); for example, the conditioned responses had a slower build-up and smaller maximum amplitude than the unconditioned responses, and the peak velocity of conditioned responses in trained animals was about five times smaller than that of unconditioned responses. Even though the unconditioned and conditioned eyelid responses showed differences in their kinetic properties, they still could be generated using the same common neural oscillator. 

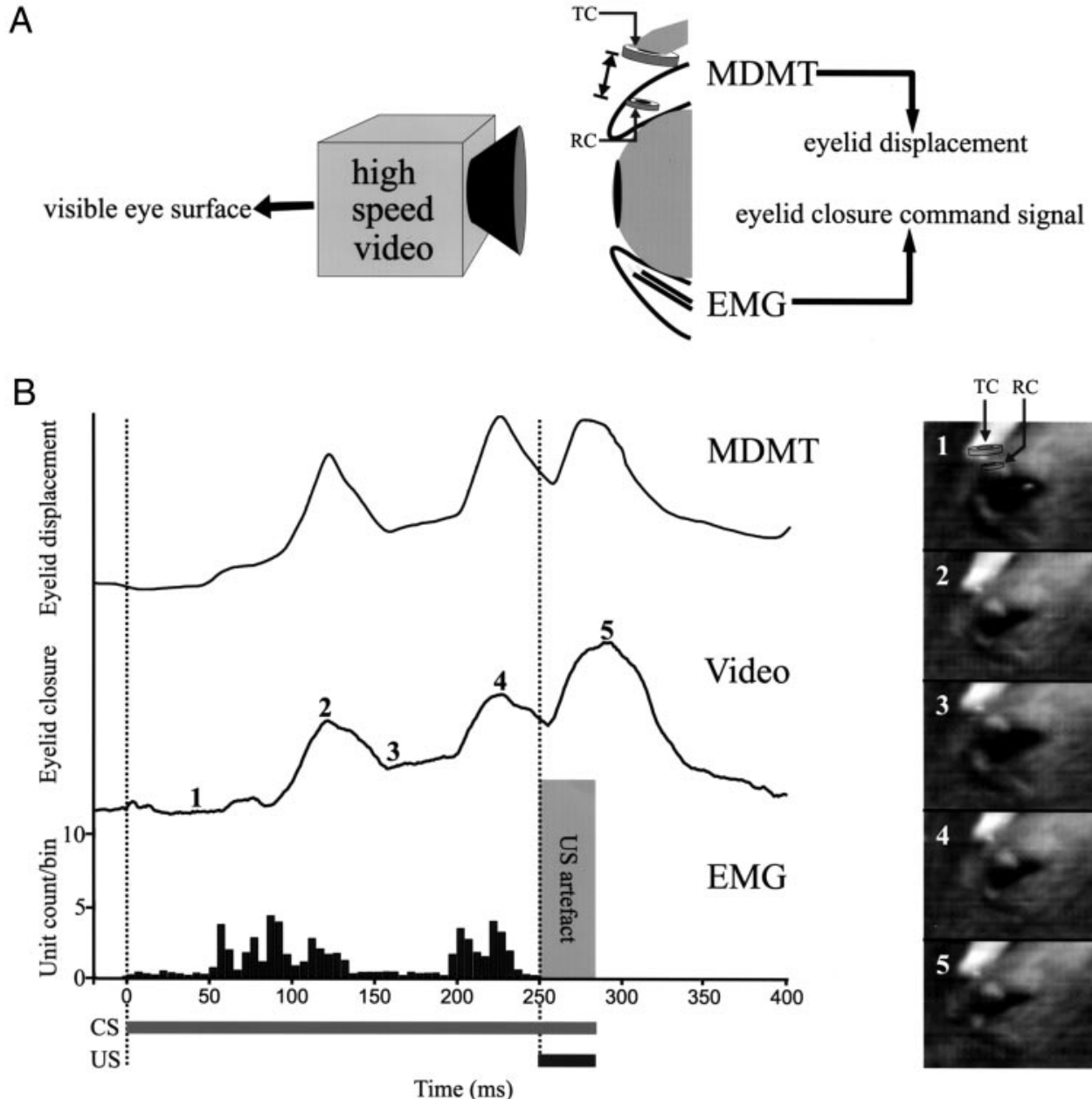

Indeed, both types of responses in mice showed oscillatory components of $\sim 25 \mathrm{~Hz}$ that were independent of the number of the training session and the ISI. Interestingly, this preferred frequency for mice is in line with an hypothesis by Gruart and others $(2000 \mathrm{a}, \mathrm{b})$ that predicts that eyelid movements have an underlying oscillator with a dominant frequency that is related to the weight of the species (Fig. 6). Thus because of the observed differences in response kinetics between unconditioned and conditioned responses, we can conclude that these responses in mice are controlled through different neural pathways, but because both response types showed similar frequency domain properties they are probably generated by a common neural oscillator.

The data presented in the preceding text are relevant because the kinetic and frequency domain properties of eyelid responses may differ between different strains of mice or different types of transgenics. Insight into these differences may for example prevent false claims about cerebellar contributions to associative learning as a particular impairment in eye-blink conditioning could be caused by a performance deficit rather than a deficit that directly and specifically affects motor learning (Steinmetz et al. 1992; Welsh and Harvey 1989). The data provided in the preceding text also indicated that the context of the experimental setup can play a role in the rate of acquisition. For example, the high-speed video recordings showed that restraining the animal impairs the acquisition of discrete eyelid conditioned responses, whereas the total number of responses

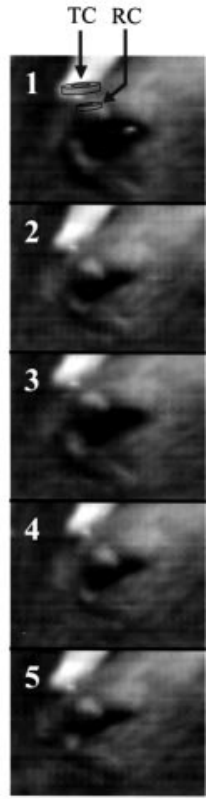

FIG. 5. High-speed Video evaluation. To evaluate the spatiotemporal accuracy of the MDMT-system and to compare this accuracy with that of the EMG system, all three methods, i.e., high-speed video, MDMT (inductive system) and EMG, were applied simultaneously in the same mice. (A) Scheme showing how the three recording methods were implemented around a single eye. $\mathrm{TC}=$ Transmitter Coil (head-fixed magnetic field generator); RC = Receiver Coil. $(B)$ Example of three simultaneous recordings with MDMT, high-speed video and EMG during a paired trial (from top to bottom). The MDMT signal closely follows that of the automated video signal in space and time (with regard to both onset and peak latency), while the converted EMG signal shows more variation in its amplitude and precedes the video signal at different latencies (MDMT peak velocity was $206 \mathrm{~ms}$, while EMG peak latency was $89 \mathrm{~ms}$ ) during the various parts of the trace. Note that the unconditioned response can be monitored successfully with the use of MDMT. Micrographs 1 to 5 in panel on the right correspond to numbers in video trace.

are increased. Because stress has been reported to influence classical conditioning, fixation stress may explain why conditioning of a freely moving mouse shows different results (Neufeld and Mintz 2001; Shors et al. 1992). Potentially, fear-related processes are able to influence classical conditioning experiments. At present it is unknown to what extent the eyelid motor system of mice in itself is involved in these fear-related processes. However, such processes may be particularly relevant in mice because the correlation coefficient of the peak amplitude - peak velocity relation of the conditioned responses was significantly lower in mice than in rabbits (Gruart et al. 2000b). In general, fear related activities may originate from direct excitatory projections from the amygdala to the eyelid motor system (Fanardjian and Manvelyan 1987) and from fear-related hormonal states, which can modulate brainstem-controlled reflexes (Lee and Davis 1997; Shors et al. 2000). Thus one should attempt to avoid potential factors that may influence fear induction during eye-blink-conditioning experiments in mice.

With the use of MDMT recordings in cerebellar mutants, we will now be able to investigate the cellular mechanisms underlying eyelid motor performance and to properly dissect the molecular components of the cerebellar network that are responsible for the control of eye-blink conditioning. Because MDMT allows us to investigate the kinetics continuously over time, we can for example also start to investigate specific cerebellar conditioning hypotheses in which timing mecha- 


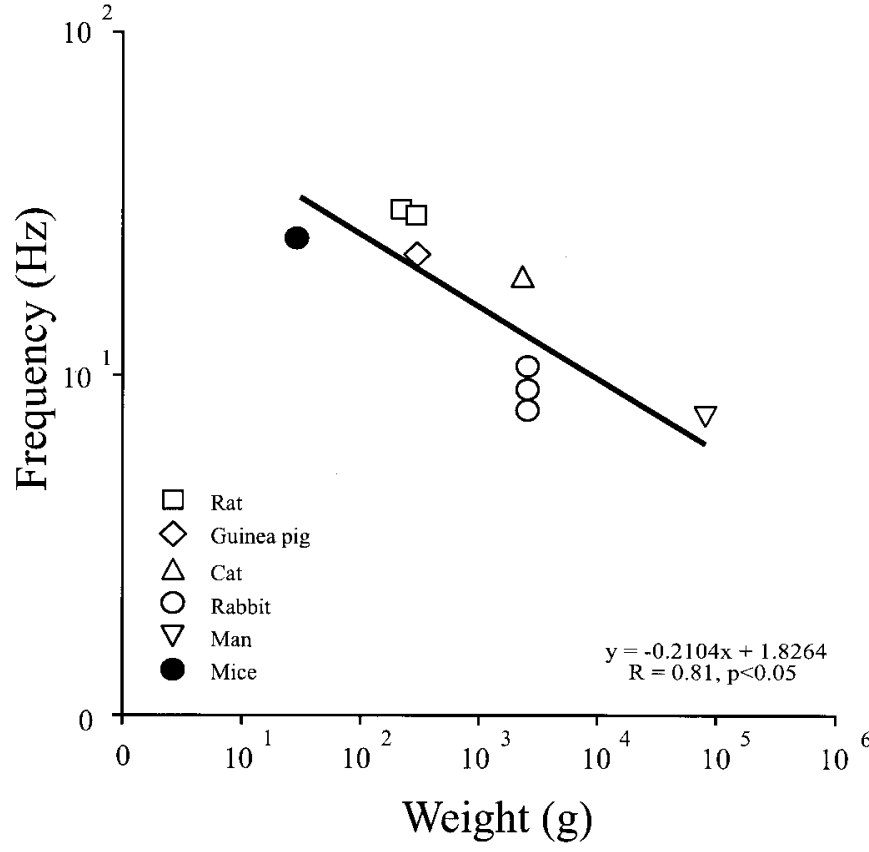

FIG. 6. Relationship between mean body weight and the dominant frequency of eyelid responses for different species. The dominant frequency of the eyelid motor system of the mouse is depicted (-) among that of other species (open symbols) as described by Gruart et al. (2000). The regression line is recalculated and the resulting equation and $\mathrm{R}$-value is shown. The fact that our data did not significantly change the correlation coefficient indicates that it is in line with the hypothesis that there is an underlying oscillator that controls eyelid movement with a frequency that can be correlated with the species mean body weight.

nisms play a dominant role (Mauk and Ruiz 1992; Mauk et al. 2000; Medina and Mauk 2000; Medina et al. 2000; Perrett et al. 1993). In addition, MDMT may provide a new methodological asset to the field of trace conditioning, in which interstimulus intervals and timing also play an important role (Buhusi and Meck 2000; Weiss and Thompson 1991).

We thank E. Dalm and H. vd Burg for technical assistance.

This study was supported by Dutch Organisation for Life Sciences (NWOALW), Dutch Organisation for Medical Sciences (NWO-MW), Human Frontier Science Program, National Institutes of Health, and European Community.

\section{REFERENCES}

Aiba A, Kano M, Chen C, Stanton Me, Fox GD, Herrup K, Zwingman TA, AND Tonegawa S. Deficient cerebellar long-term depression and impaired motor learning in mglur1 mutant mice. Cell 79: 377-388, 1994.

ARTS T AND RENEMAN RS. Measurement of deformation of canine epicardium in vivo during cardiac cycle. Am J Physiol Heart Circ Physiol 239: H432H437, 1980.

Attwell PJ, Rahman S, and Yeo CH. Acquisition of eyeblink conditioning is critically dependent on normal function in cerebellar cortical lobule hvi. J Neurosci 21: 5715-5722, 2001.

BECKER W AND FuCHS AF. Lid-eye coordination during vertical gaze changes in man and monkey. J Neurophysiol 60: 1227-1252, 1988.

Bunusi CV AND MecK WH. Timing for the absence of a stimulus: the gap paradigm reversed. J Exp Psychol Anim Behav Process 26: 305-322, 2000.

Chen C, Kano M, Abeliovich A, Chen L, Bao S, Kim JJ, Hashimoto K, THOMPSON RF, AND TONEGAWA S. Impaired motor coordination correlates with persistent multiple climbing fiber innervation in pkc gamma mutant mice. Cell 83: 1233-1242, 1995.

CHEN L, BAo S, LockARD JM, KIM JK, AND THOMPSON RF. Impaired classical eyeblink conditioning in cerebellar-lesioned and purkinje cell degeneration (pcd) mutant mice. J Neurosci 16: 2829-2838, 1996.
Conquet F, Bashir ZI, Davies CH, Daniel H, Ferraguti F, Bordi $\mathrm{F}$, Franz-Bacon K, Reggiani A, Matarese V, Conde F, Collingridge GL, AND CREPEL F. Motor deficit and impairment of synaptic plasticity in mice lacking mglur1. Nature 372: 237-243, 1994.

Delgado-Garcia JM, Evinger C, Escudero M, and BaKer R. Behavior of accessory abducens and abducens motoneurons during eye retraction and rotation in the alert cat. $J$ Neurophysiol 64: 413-422, 1990.

Disterhoft JF, Kronforst-Collins M, Oh MM, Power JM, Preston AR, AND WeISS C. Cholinergic facilitation of trace eyeblink conditioning in aging rabbits. Life Sci 64: 541-548, 1999.

Domingo JA, Gruart A, AND Delgado-Garcia JM. Quantal organization of reflex and conditioned eyelid responses. J Neurophysiol 78: 2518-2530, 1997.

Evinger C, Manning KA, and Sibony PA. Eyelid movements. Mechanisms and normal data. Invest Ophthalmol Vis Sci 32: 387-400, 1991.

FANARDJIAN VV AND MANVELYAN L. R. Mechanisms regulating the activity of facial nucleus motoneurons. III. Synaptic influences from the cerebral cortex and subcortical structures. Neuroscience 20: 835-843, 1987.

GMR TECHNOLOGY SHEET [ONLINE]. NVE Corp. http://www.nve.com/ technical/gmr/gmr.Html [2001, April 15].

Gruart A, Blazquez P, and Delgado-Garcia JM. Kinematic analyses of classically conditioned eyelid movements in the cat suggest a brain stem site for motor learning. Neurosci Lett 175: 81-84, 1994.

Gruart A, Guillazo-Blanch G, Fernandez-Mas R, Jimenez-Diaz L, and Delgado-Garcia JM. Cerebellar posterior interpositus nucleus as an enhancer of classically conditioned eyelid responses in alert cats. $J$ Neurophysiol 84: 2680-2690, 2000a.

Gruart A, Pastor AM, Armengol Ja, and Delgado-Garcia JM. Involvement of cerebellar cortex and nuclei in the genesis and control of unconditioned and conditioned eyelid motor responses. Prog Brain Res 114: 511528, 1997.

Gruart A, Schreurs BG, Del Toro ED, and Delgado-Garcia JM. Kinetic and frequency-domain properties of reflex and conditioned eyelid responses in the rabbit. $J$ Neurophysiol 83: 836-852, 2000b.

Hamiel SR, Bleicher JN, Tubach MR, and Cronan JC. Evaluation of the hall-effect sensor for determination of eyelid closure in vivo. Otolaryngol Head Neck Surg 113: 88-91, 1995.

Hesslow G AND Yeo C. Cerebellum and learning: a complex problem. Science 280: 1817-1819, 1998.

Kim JJ AND THOMPson RF. Cerebellar circuits and synaptic mechanisms involved in classical eyeblink conditioning. Trends Neurosci 20: 177-181, 1997.

Kishimoto Y, Kawahara S, Fujimichi R, Mori H, Mishina M, and Kirino Y. Impairment of eyeblink conditioning in glurdelta2-mutant mice depends on the temporal overlap between conditioned and unconditioned stimuli. Eur J Neurosci 14: 1515-1521, 2001.

KORHONEN T. Three-dimensional hall effect accelerometer for recording head movements of freely moving laboratory animals. Physiol Behav 49: 651652, 1991.

LeE Y AND Davis M. Role of the hippocampus, the bed nucleus of the stria terminalis, and the amygdala in the excitatory effect of corticotropin-releasing hormone on the acoustic startle reflex. J Neurosci 17: 6434-6446, 1997.

Mauk MD, Medina JF, Nores WL, and Ohyama T. Cerebellar function: coordination, learning or timing? Curr Biol 10: R522-525, 2000.

MAUK MD AND RUIZ BP. Learning-dependent timing of pavlovian eyelid responses: differential conditioning using multiple interstimulus intervals. Behav Neurosci 106: 666-681, 1992.

McCormick DA AND Thompson RF. Cerebellum. Essential involvement in the classically conditioned eyelid response. Science 223: 296-299, 1984.

Medina JF, Garcia KS, Nores WL, Taylor NM, and Mauk MD. Timing mechanisms in the cerebellum: testing predictions of a large-scale computer simulation. J Neurosci 20: 5516-5525, 2000.

MEDINA JF AND MAUK MD. Computer simulation of cerebellar information processing. Nat Neurosci 3 Suppl: 1205-1211, 2000.

NEUfELD M AND MinTZ M. Involvement of the amygdala in classical conditioning of eyeblink response in the rat. Brain Res 889: 112-117, 2001.

PerRetT SP, RUIZ BP, AND MaUk MD. Cerebellar cortex lesions disrupt learning-dependent timing of conditioned eyelid responses. J Neurosci 13: 1708-1718, 1993.

Phillips RG AND LeDoux JE. Differential contribution of amygdala and hippocampus to cued and contextual fear conditioning. Behav Neurosci 106: $274-285,1992$. 
QUINN KJ, KENNEDY PR, WEISS C, AND DisTERHOFT JF. Eyeball retraction latency in the conscious rabbit measured with a new photodiode technique. J Neurosci Methods 10: 29-39, 1984.

Renterghem RJ. V. Aortic Valve Geometry During the Cardiac Cycle $(\mathrm{PhD}$ thesis). Eindhoven, The Netherlands: Technical University Eindhoven, 1983.

Rodriguez F, SAlas C, VARGas JP, AND TORRES B. Eye-movement recording in freely moving animals. Physiol Behav 72: 455-460, 2001.

Schicatano EJ, Mantzouranis J, Peshori KR, Partin J, and Evinger C. Lid restraint evokes two types of motor adaptation. J Neurosci 22: 569-576, 2002.

Shibuki K, Gomi H, Chen L, Bao S, Kim JJ, Wakatsuki H, Fujisaki T, Fujimoto K, Katoh A, Ikeda T, Chen C, Thompson RF, and Itohara S. Deficient cerebellar long-term depression, impaired eyeblink conditioning, and normal motor coordination in gfap mutant mice. Neuron 16: 587-599, 1996.

Shors TJ, BEylin AV, Wood GE, AND Gould E. The modulation of pavlovian memory. Behav Brain Res 110: 39-52, 2000.
Shors TJ, WeIss C, AND Thompson RF. Stress-induced facilitation of classical conditioning. Science 257: 537-539, 1992.

Steinmetz JE, Lavond DG, Ivkovich D, Logan CG, and Thompson RF Disruption of classical eyelid conditioning after cerebellar lesions: damage to a memory trace system or a simple performance deficit? J Neurosci 12: 4403-4426, 1992

THOMPSON LT, MOYER JR JR, AND DISTERHOFT JF. Trace eyeblink conditioning in rabbits demonstrates heterogeneity of learning ability both between and within age groups. Neurobiol Aging 17: 619-629, 1996.

VAN HAM JJ AND YEO CH. Trigeminal inputs to eyeblink motoneurons in the rabbit. Exp Neurol 142: 244-257, 1996.

WeISS C AND THOMPSON RF. The effects of age on eyeblink conditioning in the freely moving fischer-344 rat. Neurobiol Aging 12: 249-254, 1991.

Welsh JP AND HARVEY JA. Cerebellar lesions and the nictitating membrane reflex: performance deficits of the conditioned and unconditioned response. J Neurosci 9: 299-311, 1989. 